\title{
Fast Track Treatment for Puberphonia
}

\author{
Kumaresan $\mathrm{M}^{*}$ and Navin Bharath \\ ENT Surgeon, Madras University, India \\ *Corresponding author: M Kumaresan, ENT Surgeon, Madras University, India
}

Received: 誹January 13, 2020

Published: 彗February 05, 2020

\begin{abstract}
Puberphonia is most often treated using voice therapy (vocal exercises) by speech-language pathologists or speech therapists that have experience in treating voice disorders. The duration of treatment is commonly one to five weeks. Indirect treatment options for puberphonia focus on creating an environment where direct treatment options will be more effective. Counseling, performed by the, a psychologist, or counselor, can help patients identify the psychological factors that contribute to their disorder and give them tools to address those factors directly. It may take long time. Patients may also be educated about good vocal hygiene and how their behavior could have long term effects on their voice. In some cases when traditional voice therapy is ineffective, surgical interventions are considered. This can occur in situations where intervention is delayed or the patient is in denial, causing the condition to become resistant to voice therapy. Surgical treatment correction needs voice therapy for a long time follow up. We use voice pitch analyzer to detect puberphonia and get the confidence of the patient. We explain the clients how by our method of pharyngeal resonance manipulation we get the male voice. By our procedure using uvula and soft palate as a source of generating male voice we eliminate high pitch voice and nasal phonation. $99 \%$ of the cases we get the male desired voice in the first instant of pharyngeal resonance manipulation. We teach breath of fire, kundali yoga to maintain the voice and make it as a habit.
\end{abstract}

Keywords: Puberphonia, speech therapy, boys talking in female tone, pharyngeal resonance manipulation, childish voice, voice breaks, feedback, genes influence in voice

Abbreviations: SRS: Session Rating Scale; ORS: Outcome Rating Scale; DNA: Deoxyribonucleic Acid; F0: Fundamental Frequency; R: Roughness; B: Breathiness; A: Asthenia; S: Strain

\section{Aim}

The aim of this article is to review the extant literature on puberphonia, stressing the significance of building confidence for effective practice and the need for more research in this area. We build the confidence for treating doctors and ailing puberphonia clients. This is achieved by foster identification, effective pharyngeal resonance manipulation treatment. Clients happy with the ancestral voice and we give a fast track package.

\section{Object}

One important objective of puberphonia treatment is to foster professional confidence and consequently competence in patient communication and clinical (physical) skills-two components vital to the profession. Professional competence can be conceptualized in terms of knowledge, abilities, skills, and attitudes displayed in the context of a set of realistic professional tasks, whereas confidence in oneself symbolizes the belief that one has the ability to do things well or deal with situations successfully. How confidence can be fostered as an integral part of puberphonia is not well known and research is still scarce. Voice therapy for puberphonia is a promising modality of treatment but not much reported in literature. Our new pharyngeal resonance manipulation in the treatment of puberphonia is similar to voice therapy. Instead of external voice therapy or external conventional laryngeal massage, we do internal pharyngeal manipulation. It is cost effective, less time-consuming procedure with no complication and gives excellent results. We do easy identification of puberphonia and get the confidence in undergoing treatment. Identification by voice pitch analyzer, get the confidence by showing the normal anatomy of their larynx by laryngeal endoscope. Puberphonia patient will understand their inappropriate use of high-pitched voice beyond pubertal age in males. It is usually seen in the immediate post-pubescent period with normal larynx [1]. 


\section{Break Through Therapy by Fast Track}

Patient feels and hears their normal low-pitched voice on the first instance of our special pharyngeal resonance manipulation. Few recognize (10\%) their ancestral tone in their new speech. Because each of these approaches implies speed, there are high success rate in treating puberphonia and the meaning of each successful case create a new life and way of life distinctions among them. An approach called informed feedback treatment or FIT does just that - uses a client's feedback to inform their treatment. FIT "is all about empowering the client and increasing the client's voice. After treatment by pharyngeal resonance manipulation in puberphonia clients get the normal voice and speech. We do a live recording their new male voice on their own cellphone and they have seen the video then and there and get a feedback and practice [2]. Specifically, FIT involves routinely and most importantly formally soliciting feedback from clients about the process of therapy, working relationship in puberphonia management and overall well-being. The formal aspect of FIT is key because most think they ask for feedback, but when they're observed live or on video, they don't do it nearly as much as they believe. Receiving ongoing formal feedback from clients has clear-cut benefits. It's been shown to boost the effectiveness of therapy, including enhancing clients' wellbeing and decreasing dropout rates and noshows. And it makes sense: Once we know precisely how the client is feeling, they're better equipped to adjust treatment accordingly.

\section{Vocal cord assessment}

These patients underwent detailed ENT evaluation by an ENT surgeon and a stroboscopic evaluation was done using Kay Pentax 9105 System. Stroboscope was used to obtain a visual assessment of the vocal cords. The stroboscopic evaluation provided measures of vibratory behavior of the vocal folds such as presence or absence of mucosal wave, vibratory symmetry, and amplitude; type of glottic closure; hyper function; arytenoids movement and symmetry; ventricular movement, etc. Stroboscope also yielded a measure of the patient's fundamental frequency (Fo) during sustained phonation.

\section{We employed fast track treatment}

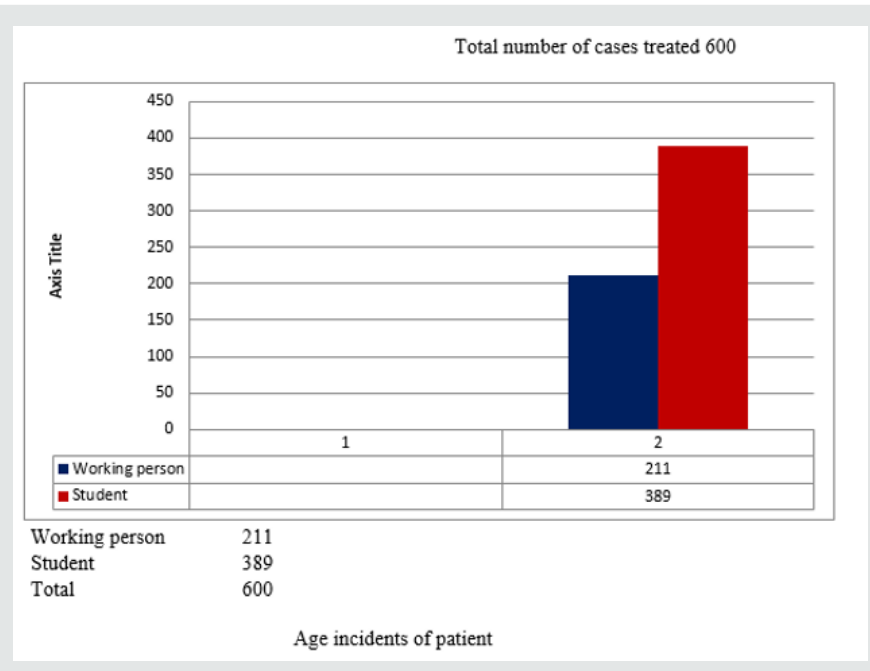

Figure 1: Age incidents of patient.

\section{Phonatotary apparatus assessment}

A perceptual assessment of patient's voice was done using the GRBAS scale. This scale consists of judgment of voice quality on the basis of Grade (G), Roughness (R), Breathiness (B), Asthenia (A), and Strain $(S)$ in voice production. The severity was graded on a 4-point rating scale, on which 3 being worst and 0 being normal [3].

\section{Treatment Methods}

The voice therapy treatment protocol included therapy techniques commonly applied for achieving lowering of pitch. Some techniques which were commonly used were as follows:

a) Humming while gliding down the pitch scale, i.e., humming while gliding from a higher note to a lower note

b) Phonation of vowel sounds with a glottal attack, i.e., forceful initiation of voice during production of vowels.

c) Use of vegetative sounds like cough or throat clear to initiate voicing.

d) Production of glottal fry (i.e., lowest possible pitch which the patient can produce).

e) Digital manipulation of thyroid cartilage during vowel production-patient is taught to apply a gentle inward push on the anterior aspect of the thyroid cartilage while sustaining a vowel.

The other treatment available is digital laryngeal manipulation, in which thyroid cartilage is being compressed, and patient is asked to speak. Later on, patient is taught to repeat this procedure at home to sustain male voice [4]. This again needs a lot of patient encouragement and follow up. No corrective surgical procedure is available except Pau H et al. [5]. who has reported first case of surgical correction of puberphonia by mobilization of hyoid and superior halves of thyroid cartilage and reducing cricothyroid distance by opposing mobile hyoid to fixed cricoid cartilage by 2 non-absorbable figure of eight sutures. 
The method reported by the author; while doing examination of the pharynx by tongue depressor or direct laryngoscope examination of a patient of puberphonia few are able to bring out normal male voice while they cough or talk. We have used a novel approach by pharyngeal resonance manipulation which gives immediate and permanent relief. We do manipulation at the level of uvula. This, our method is referred by Sudhakar Vaidya et al. [6] in his article (No reference is available except from M Kumerasan [7] (Chennai), who has published his work in book "A research work in Otorhinolaryngology" in 1992). Between August 1992 and November 2019, 600 puberphonia patients were included: The Fast Track program involved: integrated coordination between puberphonia males, their parents, friends and relatives. This procedure is done as outpatient training in hospital with faster transfer to a natural ancestral speech. Primary outcomes were functional speech status and quality of life measured through questionnaires at baseline. Outcomes were analyzed using a linear mixed-effects regression mode (Figures 1\&2).

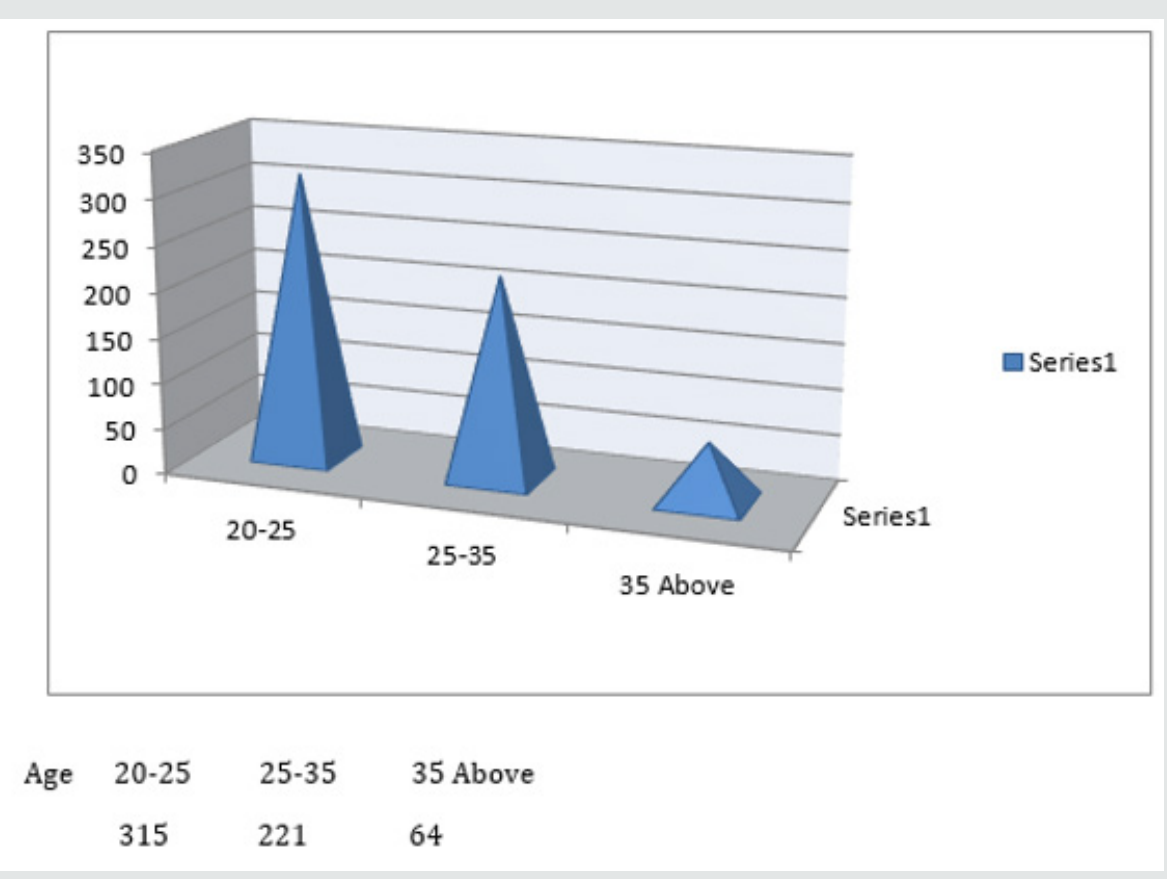

Figure 2: Outcomes were analyzed using a linear mixed-effects regression mode.

\section{Results}

The time duration of voice therapy according to Vrushali Desai et al. [8] ranged from 1 to 10 months (average-3.6 months). Pitch and quality of the voice became normal in $78.9 \%$ and $35.2 \%$ of patients respectively. It is estimated that within four weeks of therapy voice changes could be achieved. This time is shorter for subjects who present vocal hyper function. Of the same, the subjects without hyper function could require more time of therapy. Regarding the duration of therapeutic progress, there are studies that make between 6 and 24 months of follow-up after speech therapy, maintaining frequency values. Regarding the choice of approach for puberphonia, most of the reviewed studies agree that the best therapeutic option is to start with speech therapy and if this type of therapy does not provide positive results it is possible to seek a surgical option. On the other hand, the investigations reveal that the therapy is very efficient in relation to the number of sessions, since in the same month it would be possible to obtain consistent changes in the voice. We do" Pharyngeal resonance manipulation" at the first instances itself and brings the desired low-pitched voice. We train them for three days by practicing breath of fire yoga and make it as a habit by doing the same yoga for 21 days [9].

\section{Follow up by feedback method}

Two of the most popular measures of feedback [10] are the Outcome Rating Scale (ORS) and the Session Rating Scale (SRS). The ORS, which a client completes at the start of a session, asks about their wellbeing. The SRS, which is filled out at the end, asks about our performance. For instance, one item asks if the client felt heard, understood and respected during the session. Another asks if they worked on or talked about what they wanted to. It had a huge impact on their improvement. Feedback was especially critical for clients who had a huge impact on their improvement. Feedback was especially critical for clients who weren't getting better, since this group tends to leave therapy early. Seeing and getting their desired tone in their voice even found a boost in retention rates. We found that clients in the feedback condition showed about twice as much improvement as clients who didn't provide feedback and in fewer sessions. We found that using feedback measures leads to fewer noshows and dropouts. The results of the technique were grouped into excellent, satisfactory and poor. The success rate of direct laryngeal manipulation in first sitting was $99 \%$. Puberphonia is not a rare disorder. Even though we have found our own simple method of puberphonia relief in 1990 [7]. Vigorous propaganda is done since 
11 November 2016. In a short period in one center we got 600 till date $(28 / 01 / 2020)$ cases indicates that prevalence of puberphonia is high. Apart from impact on voice of patient, puberphonia also has impact on the psycho-social aspect.

\section{Discussion}

\section{Professional confidence in puberphonia management}

In health care there is a complex interaction between provider and patient; this remains at the very core of the healing process and has always had a pronounced impact on patient health and recovery. The complexity of this interaction has historically distinguished the puberphonia practice profession from the rest of the ENT, medical, problems. Confidence, defined as a belief that one will act in an effective way, is expected to play a critical role in how a clinician makes decisions, utilizes his or her skills, communicates with patients, treat and get the desired result. Puberphonia treatment needs much more than that because it reflects client's self-esteem and future life. Yes $99 \%$ of future life depends on their voice.

\section{Voice and genes relationship}

The human voice has appreciated that the quality of the voice influenced the society in big scale. While on conversation whether male or female usually notice their pitch quality, quality of the voice, phonation style and so on Also when same person talks again over phone many people recognize their voice by their quality of the voice. Many researchers found that the relationship of the voice quality and genes play a vital role in determining their offspring's voice [11]. Although voice production is a complex functioning of much system, we can see the voice resemblance runs in many families. In the family when the baby born people will be very happy and explore the features of the baby of its resemblances of the nose, ear, hair ,facial appearance and so on. In addition, in their family the elders will compare and recognize the baby's appearance and resemblances of their ancestor. Simply because these variability's run in their family, since, these will be written in the Gene (DNA). Similarly, we can see not only their ancestor's features and also their voice also written in the gene (DNA). In particular, for males, during their puberty voice change occur, that time only people recognize and compare young adult voice with their father or forefather voice.

\section{Genes influence on the voice}

Genes are segments of Deoxyribonucleic acid (DNA) that contains the code for specific protein that functions one or more types of cells in the body. The chromosomes are structure within the cell that contains person's genes. Researcher revealed that the human has Forehead box protein P2 is a protein that, in humans, is encoded by the FOX2 gene [12] also known as CAGH44, SPCH1and is required for proper development of speech and language. FOXP2 is a transcription factor, meaning that it encodes for a regulatory protein. The gene shared with many vertebrates ,and it generally play a role of communication the other species Chimpanzees are also found FOXP2 gene, but it had some mutational changes and they differ than human version. In human FOXP2 gene is located on the arm of chromosome 7 at position. So, with this evidence, it clearly denotes that the genes are segment of deoxyribonucclic acid
(DNA) and it play a role in how our voice determination. Although how a child's voice develops owes something to mimicry of their parents, people from the same family will often sound alike because laryngeal anatomy is dictated by your ancestral DNA just like every other physical trait. It's the slight variations around this anatomy that make our voices distinct. Gene factors cause variations between members of the same sex with males and females and voice being categorized in the voice type. Actually, voice qualities are largely determined by the size and shape of the larynx, neck, throat and facial structures all determined by genetics.

\section{Relationship of ancestral voice and gene}

In our hospital we are correcting Puberphonia client's voice (female) by using Resonance Manipulation Therapy treatment method. In that we selected 600 cases and did research of the voice and the genes influence in their Ancestors by using convenient sampling technique .At the time of the training time many have exclaimed that their voice resembles their father's voice, some claimed as uncle's voice and few claimed grandfather's voice and many could not recognize their voice quality. This may be due to or probably in our family system has changing from joint family to nuclear family system. Secondly gene influence on voice may be their fore father and people cannot recognize, because they are not existing now (Table 1). The table clearly denotes that among 600 samples $90(16 \%)$ had their father's voice,58(9.6\%) had uncle's voice, $32(6.4 \%)$ resumed grandfather's voice and 340 clients not known their voice quality their voice. This may be probably because of our new mutated family system and our forefather's voice may not know by existing generation [13].

Table 1: Genes Influence in Voice Quality.

\begin{tabular}{|c|c|c|c|}
\hline S. No & Voice relationship & nos & Percentage \% \\
\hline 1 & Father's voice & 90 & 16 \\
\hline 2 & Uncle's voice & 58 & 9.6 \\
\hline 3 & Grandfather's voice & 32 & 6.4 \\
\hline 4 & Un-recognized voice & 340 & 68 \\
\hline 5 & Total samples & 600 & 100 \\
\hline
\end{tabular}

\section{Conclusion}

Acoustic Analysis: Fundamental Frequency (F0) was assessed pre-and post-therapy for all the patients. The average value of F0 before therapy was $208 \mathrm{~Hz}$, and following therapy, it lowered to an average of $125 \mathrm{~Hz}$, which was a significant improvement.

Stroboscopic Findings: of the 600 patients,500 had normal stroboscopic evaluation. Seven of the 100 patients had minimal phonatory Gap as the only clinical finding. The remaining stroboscopic parameters were normal. Since the pre-therapy findings were not clinically significant, the stroboscopy was not repeated during the follow-up.

Perceptual Ratings: The pre-therapy perceptual ratings revealed mild deviance in overall grade, asthenia, and breathiness. The post-therapy ratings showed improvement to a "normal" overall rating with no perceptual evidence of breathiness and asthenia. The pre-therapy high-pitched voice had lowered to a normal pitch 
range at the end of therapy. Puberphonia fast track identification, treatment, building confidence and care as habituation programs were effective in that multi-trauma patients improved their functional status and quality of life. A faster (maximum) recovery in functional status was observed for fast track at 1st day compared to other modalities of treatment. At twelve months follow-up no differential effects between treatment conditions were found.

\section{References}

1. Matthew DS, Elisa MT, Donna LC, Criag RC (2016) Selection and Socialization Effects in Early Adolescent Alcohol Use: A Propensity Score Analysis. Journal of Abnormal Child Psychology 43(6): 1131-1143.

2. Delgadillo J, de Jong K, Lucock M, Lutz W, Rubel J, et al. (2018) Feedbackinformed treatment versus usual psychological treatment for depression and anxiety: a multisite, open-label, cluster randomised controlled trial. The Lancet Psychiatry 5(7): 564-572.

3. Katia Nemr, Marcia Simões Zenari, Gislaine Ferro Cordeiro, Domingos Tsuji, Allex Itar Ogawa, et al. (2012) GRBAS and Cape-V Scales: High Reliability and Consensus When Applied at Different Times, Journal of voice 26(6): 812e17-812e22.

4. Kizilay A, Firat Y (2008) Treatment algorithm for patients with puberphonia. Kulak Burun Bogaz Ihtisas Dergisi 18(6): 335-342.
5. Pau H, Murty GE (2001) First case of surgically corrected puberphonia. The Journal of Laryngology \& Otology 115(1): 60-61.

6. Sudhakar Vaidya, G Vyas (2006) Puberphonia: A novel approach to treatment. Indian Journal of Otolaryngology and Head and Neck Surgery 58(1): 20-21.

7. Kumaresan M (1992) Clinical and Practical Otorhinolaryngology: A Research work in Otorhinolaryngology. ( $1^{\text {st }}$ edn $)$, Paramkalyan printers, Madras, India.

8. Vrushali Desai, Prasun Mishra (2012) Voice therapy outcome in puberphonia. Journal of Laryngology \& Voice 2(1): 26-29.

9. Muthiah K (2018) An immediate and permanent cure for Puberphonia. J Otolaryngol ENT Res 10(4): 231.

10. Bovendeerd B, de Jong K, Colijn S (2019) Systematic client feedback to brief therapy in basic mental healthcare: study protocol for a four-center clinical trial. BMJ Open 9(5): e025701.

11. Robert Thayer Sataloff (1995) Genetics of the voice. Journal of Voice $9(1): 16-19$.

12. Wolfgang Enard (2002) Molecular evolution of FOXP2, a gene involved in speech and language. Nature 418(6900): 869-872.

13. Margaret Fawcus (1991) Voice Disorders and their Management, Springer.
(C) This work is licensed under Creative

To Submit Your Article Click Here: Submit Article

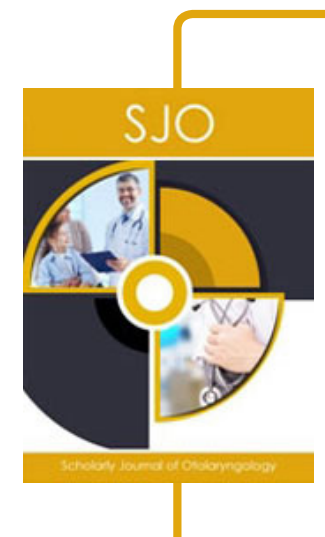

Scholarly Journal of Otolaryngology

\section{Assets of Publishing with us}

- Global archiving of articles

- Immediate, unrestricted online access

- Rigorous Peer Review Process

- Authors Retain Copyrights

- Unique DOI for all articles 